\title{
Central Respiratory Rhythmogenesis Is Abnormal in Lbx1- Deficient Mice
}

\author{
Silvia Pagliardini, ${ }^{1 \star}$ Jun Ren, ${ }^{1 \star}$ Paul A. Gray, ${ }^{2}$ Cassandra VanDunk,,${ }^{2}$ Michael Gross, ${ }^{3}$ Martyn Goulding,, 3 and \\ John J. Greer ${ }^{1}$ \\ ${ }^{1}$ Department of Physiology, Centre for Neuroscience, University of Alberta, Edmonton, Alberta, Canada T6G 2S2, ${ }^{2}$ Department of Anatomy and \\ Neurobiology, Washington University Medical School, St. Louis, Missouri, 63110-1093, and ${ }^{3}$ Molecular Neurobiology Laboratory, The Salk Institute for \\ Biological Studies, La Jolla, California 92037
}

\begin{abstract}
$L b x 1$ is a transcription factor that determines neuronal cell fate and identity in the developing medulla and spinal cord. Newborn $L b x 1$ mutant mice die of respiratory distress during the early postnatal period. Using in vitro brainstem-spinal cord preparations we tested the hypothesis that $L b x 1$ is necessary for the inception, development and modulation of central respiratory rhythmogenesis. The inception of respiratory rhythmogenesis at embryonic day 15 (E15) was not perturbed in $L b x 1$ mutant mice. However, the typical age-dependent increase in respiratory frequency observed in wild-type from E15 to P0 was not observed in $L b x 1$ mutant mice. The slow respiratory rhythms in E18.5 Lbx1 mutant preparations were increased to wild-type frequencies by application of substance P, thyrotropin releasing hormone, serotonin, noradrenaline, or the ampakine drug 1-(1,4-benzodioxan-6-yl-carbonyl) piperidine. Those data suggest that respiratory rhythm generation within the pre-Bötzinger complex (preBötC) is presumably functional in $L b x 1$ mutant mice with additional neurochemical drive. This was supported by anatomical data showing that the gross structure of the preBötC was normal, although there were major defects in neuronal populations that provide important modulatory drive to the preBötC including the retrotrapezoid nucleus, catecholaminergic brainstem nuclei, nucleus of the solitary tract, and populations of inhibitory neurons in the ventrolateral and dorsomedial medullary nuclei. Finally, we determined that those defects were caused by abnormalities of neuronal specification early in development or subsequent neuronal migration.
\end{abstract}

Key words: preBötzinger complex; transcription factor; apnea; medulla; fetal; Bötzinger

\section{Introduction}

Lbx1, the vertebrate homolog of the Drosophila ladybird gene (Jagla et al., 1995; Dietrich et al., 1998) is expressed in developing skeletal muscle and nervous system. This transcription factor is an important determinant of spinal dorsal neurons and somatosensory neurons in the hindbrain (Gross et al., 2002; Müller et al., 2002; Sieber et al., 2007). In the spinal cord, the inactivation of Lbx1 alters the developmental program resulting in $L b \times 1$ deficient spinal neurons acquiring a more dorsally derived commissural interneuron phenotype (Gross et al., 2002; Müller et al., 2002). Furthermore, Lbx1 is expressed by both excitatory glutamatergic and inhibitory GABAergic/glycinergic neurons in the embryonic spinal cord (Cheng et al., 2005). The loss of Lbx1 selectively eliminates these inhibitory neurons. Sieber et al. (2007) recently described $L b x 1$-derived neurons as a major com-

Received April 15, 2008; revised Sept. 12, 2008; accepted Sept. 15, 2008

S.P. is a Fellow of the Alberta Heritage Foundation for Medical Research (AHFMR) and the Canadian Institute for Health Research. J.J.G. is a Scientist of the AHFMR. We thank Qiufu Ma for the SIc6a5 probe.

*S.P. and J.R. contributed equally to this work.

Correspondence should be addressed to John J. Greer at the above address. E-mail: John.Greer@ualberta.ca.

S. Pagliardini's present address: System Neurobiology Laboratory, Department of Neurobiology, University of California, Los Angeles, Los Angeles, CA 90095.

M. Gross' present address: Department of Biochemistry and Biophysics, Oregon State University, Corvallis, OR 97331.

DOI:10.1523/JNEUROSCI.1648-08.2008

Copyright $\odot 2008$ Society for Neuroscience $\quad$ 0270-6474/08/2811030-12\$15.00/0 ponent of the spinal trigeminal tract $(\mathrm{SpV})$ in the brainstem. Here, our analysis of the brainstem revealed a wider distribution of Lbx1-derived neurons in the medulla, including in major respiratory nuclei described in this study.

Lbx1 mutant mice die shortly after birth from apparent respiratory distress. In this study, we tested the hypothesis that the breathing abnormality arises from defective central respiratory drive. Specifically, we used in vitro preparations to determine whether $L b x 1$ expression is necessary for the inception and development of rhythmic respiratory activity within the pre-Bötzinger complex (preBötC). Furthermore, we assessed the importance of Lbx1 expression for the development of medullary networks that modulate central respiratory rhythmogenesis. Neurotransmitter receptor agonists, antagonists and modulators were administered to assess the ability of neuromodulatory systems to normalize respiratory rhythmogenesis in mutant mice.

Anatomical studies using a combination of markers (transcription factors, neurotransmitter synthesizing enzymes, and peptide receptors) were then performed to determine whether there are structural abnormalities within pontomedullary structures that could account for the respiratory phenotype identified by electrophysiological recordings. This includes the preBötC, retrotrapezoid nucleus (RTN), catecholaminergic brainstem nuclei, nucleus of the solitary tract (NTS), and populations of inhibitory neurons in the ventrolateral and dorsomedial medullary nuclei. 
A

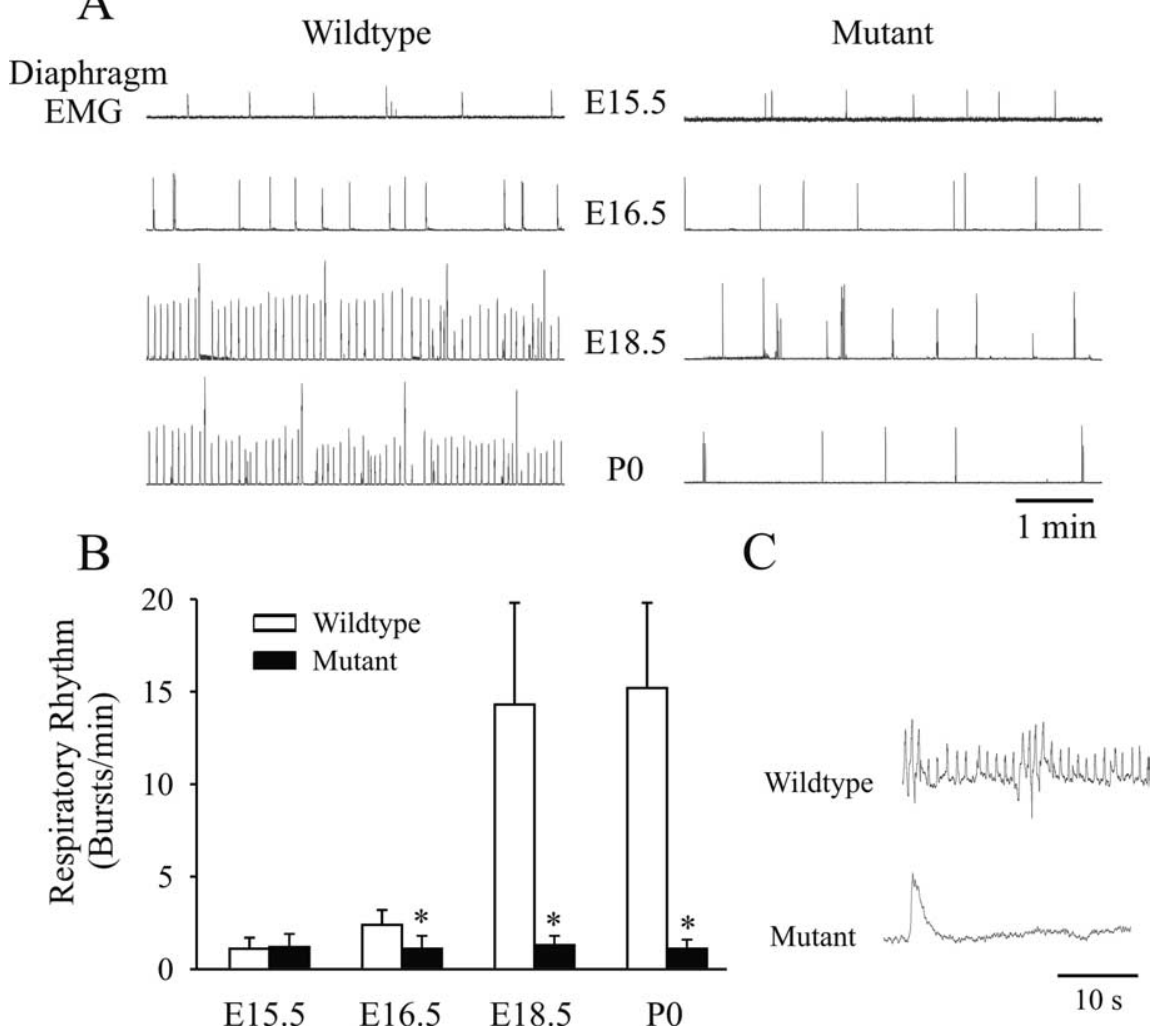

Figure 1. Respiratory rhythmic activity generated by perinatal wild-type and $L b \times 1$ mutant mice. $A$, Rectified and integrated EMG recordings from the diaphragm in brainstem-spinal cord-diaphragm preparations isolated from Lbx1 mutant and wildtype mice of different perinatal stages. The frequency of rhythmic respiratory discharge increased with age in wild-type preparations but remained relatively slow and unstable in $L b x 1$ mutant preparations. Population data were summarized in $\boldsymbol{B}$. Each data point was from 5, 6, 21, and $4 \mathrm{Lbx} 1$ mutants, 5, 6, 16, and 4 wild-type mice for E15.5, E16.5, E18.5, and P0, respectively. ${ }^{*} p<0.05$ between $L b \times 1$ mutant and wild-type of the same age group. C, Plethysmographic recordings of newborn $L b \times 1^{+/+}$and $L b \times 1^{G F P /+}$ mice.

\section{Materials and Methods}

Generation and genotyping of mice. The generation of $L b \times 1^{G F P(n e o)}$ has been described previously (Gross et al.,2002). Identification of mutant offspring was performed by PCR genotyping of snap frozen tissue with the specific primers for $L b x 1$ (MKG396, CAG CTG CAG AAG CCA GGA CTG; MKG321, CCG GAC ACG CTG AAC TTG TGG; MKG333, ATG ACT TCC AAG GAG GAC GGCA), green fluorescent protein (GFP)/ yellow fluorescent protein (YFP) (GCA CGA CTT CTT CAA GTC CGC CAT GCC; GCG GAT CTT GAA GTT CAC CTT GAT GCC, 280 bp), Amplification of mutant and wild-type $L b \times 1$ alleles generated diagnostic bands of 315 and 445 bp, respectively.

Brainstem-spinal cord preparations. Newborn mouse pups (within 15 min of birth) were anesthetized with metofane. Prenatal mice at embryonic day 15.5 (E15.5)-E18.5 were delivered from timed-pregnant mice anesthetized with halothane and maintained at $37^{\circ} \mathrm{C}$ by radiant heat. All procedures used in this study were approved by the Animal Welfare Committee at the University of Alberta. Newborn pups and embryos were decerebrated and the brainstem-spinal cord with the diaphragm muscle attached was dissected following procedures similar to those established for perinatal rats (Smith et al., 1990; Greer et al., 1992). The neuraxis was continuously perfused at $27 \pm 1^{\circ} \mathrm{C}$ (perfusion rate $5 \mathrm{ml} /$ min, chamber volume of $1.5 \mathrm{ml}$ ) with Kreb's solution that contained (in mM): $128 \mathrm{NaCl}, 3.0 \mathrm{KCl}, 1.5 \mathrm{CaCl}_{2}, 1.0 \mathrm{MgSO}_{4}, 24 \mathrm{NaHCO}_{3}, 0.5$ $\mathrm{NaH}_{2} \mathrm{PO}_{4}$, and 30 D-glucose equilibrated with $95 \% \mathrm{O}_{2} / 5 \% \mathrm{CO}_{2}, \mathrm{pH} 7.4$.

Recording and analysis. Recordings of $\mathrm{C} 4$ ventral roots and diaphragm electromyogram (EMG) were made with suction electrodes. Signals were amplified, rectified, low-pass filtered and recorded on computer using an analog-digital converter (Digidata 1200; Molecular Devices) and data acquisition software (Axoscope; Molecular Devices). Mean values rela- tive to control for the period of respiratory motoneuron discharge were calculated from a minimum of 30 consecutive bursts. Values given are means and SDs. Statistical significance was tested using one way ANOVA with HolmSidak test; significance was accepted at $p$ values lower than 0.05 .

Plethysmographic measurements. Wholebody plethysmographic measurements of the frequency and depth of breathing were made from unrestrained newborn mice using a pressure transducer (model DP 103; Validyne) and signal conditioner (CD-15; Validyne).

Pharmacological agents. All drugs were purchased from Sigma. The ampakine CX546 was dissolved in dimethylsulfoxide to make a 50 200 mM stock solution.

Animal handling for anatomical studies. Timed pregnant mice at embryonic ages (E) 10 to E18 were anesthetized with halothane $(1.5 \%$ delivered in $95 \% \mathrm{O}_{2}$ and $5 \% \mathrm{CO}_{2}$ ) and fetal mice were delivered and fixed by immersion in $4 \%$ paraformaldehyde in phosphate buffer $(\mathrm{PB})$ at pH $7.2(<\mathrm{E} 15)$. Postnatal mice were anesthetized by hyperthermia. Mice older than E15 were transcardially perfused with the same fixative solution. Brainstems were dissected and postfixed before sectioning with a vibratome (VT1000S; Leica) or cryoprotected and sectioned on a cryostat (Bright Instruments). All procedures used in this study were approved by the Animal Welfare Committee at the University of Alberta or Washington University in St. Louis.

Immunohistochemistry. Mutant and wildtype mice within the same litter were processed together for comparisons. Immunohistochemical detection of GFP with a specific antibody improved the resolution for the detection of GFP-expressing $\left(\mathrm{GFP}^{+}\right)$cells (Gross et al., 2002). Detailed protocols for the immunohistochemistry experiments have been reported previously (Pagliardini et al., 2003). In brief, transverse vibratome $(50 \mu \mathrm{m})$ or cryostat $(20 \mu \mathrm{m})$ sections were incubated with $1.0 \%$ BSA or $10 \%$ normal horse serum and $0.2-0.3 \%$ Triton X-100 in PBS for $60 \mathrm{~min}$ and then incubated overnight with primary antibodies diluted in PBS, $0.1 \%$ BSA and $0.2-0.3 \%$ Triton X-100. Primary antibodies used for this study were as follows: goat anti-choline acetyl transferase (ChAT; 1:300, Millipore Bioscience Research Reagents), rabbit antisomatostatin (SST; 1:1000, Immunostar), rabbit anti-green fluorescent protein (GFP; 1:500, Invitrogen), chicken anti-GFP (1:500, Aves Labs), rabbit anti-neurokinin 1 receptor (NK1R; 1:1000, Advance Targeting System), rabbit anti-paired homeobox domain transcription factor 2 (Pax2, 1:500, Zymed Labs/Invitrogen), rabbit anti-tyrosine hydroxylase (TH; 1:2000, Millipore Bioscience Research Reagents), rabbit antiwinged helix/Forkhead transcription factor P2 (FoxP2; 1:700, AbCam), guinea pig anti-LIM homeodomain transcription factor $1 \mathrm{~b}$ (Lmx1b; 1:500, kindly provided by Dr. T. M. Jessell, Columbia University, New York, NY), and rabbit anti-paired-like homeobox 2b (Phox2b; 1:500, kindly provided by Dr. J. F. Brunet, CNRS, Paris, France).

The following day, sections were washed and incubated with specific secondary antibodies diluted in PBS and 0.1\% BSA for $2 \mathrm{~h}$ (Cy3-, Cy5- or Cy2-conjugated donkey anti-rabbit, donkey anti-goat, donkey antiguinea pig; 1:200; Jackson ImmunoResearch; or Alexa488-conjugated anti-rabbit, donkey anti-chicken; 1:1000, Invitrogen). Sections were further washed, mounted, and coverslipped.

In situ hybridization for GlyT2. Slides are immersed in 4\% PFA, permeablized with proteinase $\mathrm{K}$, washed in $0.1 \mathrm{M}$ triethanolamine- $\mathrm{HCl}$ with $0.25 \%$ acetic anhydride, blocked in hybridization buffer at $65^{\circ} \mathrm{C}$, then placed into slide mailers containing hybridization buffer with DIG- 
labeled antisense RNA at $1 \mu \mathrm{g} / \mathrm{ml}$ overnight at $65^{\circ} \mathrm{C}$. Slides are washed in SSC buffers at $62^{\circ} \mathrm{C}$. Slides are washed and incubated in alkaline phosphatase conjugated anti-DIG antibody in $10 \%$ NHS and then incubated in NBT-BCIP until cellular labeling is clear. For combined immunohistochemistry and in situ hybridization, slides are stained for mRNA expression before immunohistochemical labeling.

Bright-field and confocal imaging. Fluorescently immunostained sections were examined and processed using a Zeiss $100 \mathrm{M}$ microscope, LSM510 NLO laser, and LSM510 software (Zeiss) or an Improvision OptiGrid structured illumination confocal using a Photometrics HQ2 camera on a Nikon 90i microscope with Phylum Software (Improvision). Thin sections and multiple sectioning acquisitions along the z-plane were performed to obtain a suitable signal through the depth of the section. Combined alkaline phosphatase in situ hybridization and fluorescently immunostained sections were acquired in bright field and then fluorescence. Acquired images in JPEG format were then exported to Photoshop for figure composition and preparation.

Surface areas of vagus $(\mathrm{X})$, nucleus ambiguus (NA) and hypoglossal (XII) nuclei were measured bilaterally for each section and an average area was calculated for each animal using LSM510 software. Noradrenergic cells immunolabeled for TH were counted in serial sections at E18 and an average and SD of cells/ nucleus calculated. Paired t tests comparing $L b \times 1^{G F P / G F P}$ mice to $L b \times 1^{G F P /+}$ litter mates were applied to determine statistical significance at $p<0.05$.

\section{Results}

Respiratory rhythm generation by wildtype and $L b x 1$ mutant in

\section{vitro preparations}

In preliminary experiments, newborn mouse pups were monitored immediately after delivery. All $L b x 1^{\text {GFP/GFP }}$ mice displayed both abnormal limb morphology and profound apnea; alternatively referred to as $L b x 1$ mutants in the paper. Previous studies have demonstrated that the expression of one copy of $L b \times 1$ gene is sufficient to promote normal embryonic development (Gross et al., 2002; Müller et al., 2002). Consistent with this, we did not find any differences in the respiratory patterns generated by $L b x 1^{+/+}$and $L b x 1^{G F P /+}$ mice. $L b x 1^{G F P /+}$ mice were used as controls for this study and are also referred to as wild-type in the remainder of the paper. To determine whether the respiratory phenotype was caused by abnormal central respiratory drive, the brainstem-spinal cords with diaphragm muscle still attached were isolated from wild-type and mutant preparations. Figure $1 \mathrm{~A}$ shows representative diaphragm EMG recordings obtained in the perinatal period, between E15.5 and postnatal day (P0). Both mutant and wild-type preparations commenced generating respiratory activity in vitro at E15. Furthermore, there were no differences in the frequency of respiratory discharge in vitro at that age. However, the frequency of respiratory rhythm was much slower in mutants relative to wild-type preparations after E15.5
Wildtype

Mutant

\section{I: BSSCD with Pons Attached}

\section{II: BSSCD as Control}
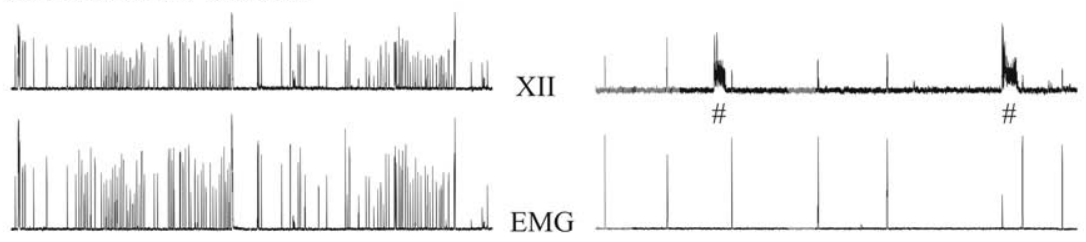

EMG

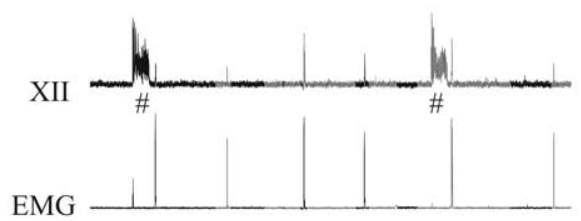

EMG

III: BSCCD without Rostral Medulla

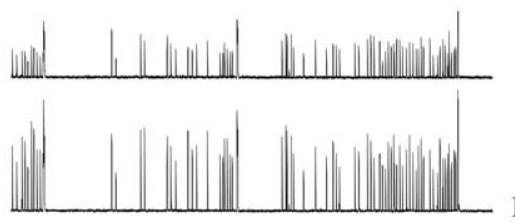

XII
EMG
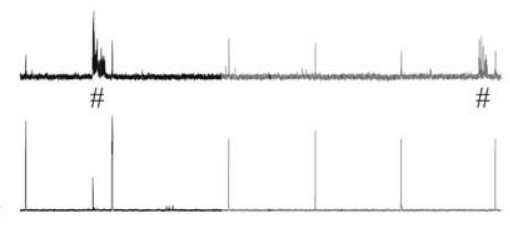

$30 \mathrm{~s}$

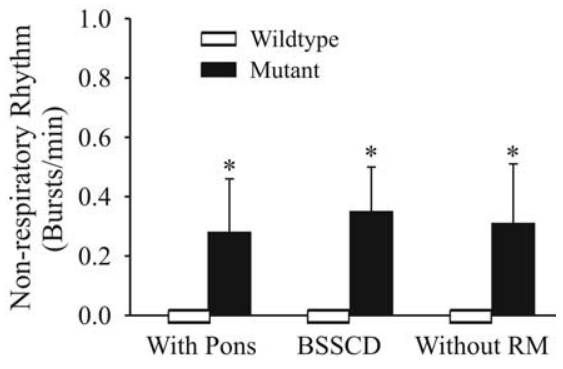

Figure 2. Lbx1 mutant in vitro brainstem-spinal cord preparations generated nonrespiratory rhythmic neural activity at E18.5. $A$, Rectified and integrated suction electrode recordings of diaphragm EMG and hypoglossal nerve root activity (XII) in brainstemspinal cord-diaphragm preparations (BSSCD) isolated from E18.5 wild-type and Lbx1 mutant mice. Recordings were from prepins with (1) pons intact, (2) medulla intact and (3) without the rostral medulla. After removal of the pons, the rhythmic wild-type preparations was not significantly altered after removal the rostral medulla (RM). The motor discharge was markedly in all Lbx1 mutant preparations, without a significant change in frequency, amplitude, duration, or regularity upon duration, nonrespiratory rhythm (\#), that was not observed in wild-type preparations at E18.5. B, Population data showing the frequency of both respiratory and nonrespiratory rhythm for each type of brainstem-spinal cord-diaphragm preparation. Each data point was from four to five preparations tested. ${ }^{*} p<0.05$, compared with wild-type.

(Fig. $1 B$ ). Population data show interburst intervals of $3.9 \pm 1.2 \mathrm{~s}$ $(n=4)$ and $55 \pm 25 \mathrm{~s}(n=4)$ in wild-type and mutant P0 preparations. Figure $1 C$ shows representative traces of plethysmographic recordings of newborn $L b x 1^{+/+}$and $L b \times 1^{G F P /+}$ mice.

All subsequent recordings were performed from in vitro preparations isolated from E18.5 mice delivered via caesarian section. This negated any potentially confounding problems associated with measurements from mutant newborn mouse preparations that may have had altered CNS function because of the hypoxia associated with postnatal hypoventilation.

A total of 45 Lbx 1 mutant and 39 wild-type mice were subsequently selected for detailed analyses. For the first studies of E18.5 mice, the respiratory rhythm was monitored using brainstemspinal cord preparations with the diaphragm muscle attached. Respiratory rhythmic bursting was apparent in population recordings of XII nerve roots and diaphragm EMG recordings in 
those preparations. The mean interval between inspiratory bursts was $4.2 \pm 1.6 \mathrm{~s}(n=16)$ in wild-type and $46 \pm 18 \mathrm{~s}(n=21)$ in Lbx1 mutant preparations. In addition to the respiratory activity, a second longer-duration ( $>3 \mathrm{~s}$ ) bursting activity was prominent on XII nerve recordings in all E18.5 Lbx1 mutant preparations (Fig. 2). This type of nonrespiratory rhythm was seldom observed in wild-type preparations at the same stage of development. The longer-duration, nonrespiratory rhythm had the same characteristics described previously for fetal mice and rats (Greer et al., 1992a; Pagliardini et al., 2003; Ren and Greer, 2003; ThobyBrisson et al., 2005). These spontaneously generated fetal rhythms are typically generated throughout the neuraxis at earlier stages of rodent development but cease occurring by E16 in mice (Thoby-Brisson et al., 2005).

These data demonstrate that $L b \times 1$ mutant mouse preparations generate a very slow rate of inspiratory bursting, which in vivo would cause rapid hypoxia and inability to survive. Although, the basic inspiratory rhythm is thought to be generated within the preBötC, neurons in that region receive synaptic input and interact with respiratory nuclei in the pons, including the A5 noradrenergic group. The parafacial respiratory group (pFRG) is also thought to be coupled with and modulate the activity of the preBötC (Onimaru and Homma, 2003; Dutschmann et al., 2004). We therefore performed a series of experiments recording respiratory discharge in $L b \times 1$ mutant preparations before and after removal of the pons and rostral medulla to test whether those structures were contributing to the abnormal respiratory rhythm in vitro.

Figure $2 A$ shows examples of recordings from each type of preparation derived from wild-type and $L b x 1$ mutant preparations. Past reports state that E18.5 mouse brainstem-spinal cord preparations typically fail to generate a respiratory rhythm with the pons intact (Viemari and Gérard, 2002). However, all of our E18.5 wild-type brainstem-spinal cord-diaphragm preparations with the pons attached generated a robust stable rhythm with a frequency of $5.4 \pm 3.4$ bursts/min $(n=4)$. The removal of the pons in wild-type preparations resulted in the coefficient of variability increasing more than twofold $(0.53 \pm 0.10$ with pons vs $1.22 \pm 0.14$ without pons, $n=5)$ and overall bursting frequency increasing to $16.9 \pm 6.7$ bursts/min $(n=5)$ (population data is shown in Fig. $2 B$ ). With the pons removed, the characteristic periodic pattern of motor discharge often seen in mouse, but only occasionally in rat preparations, emerged. Specifically, a train of shorter duration bursts was followed by a single burst of larger amplitude and longer duration which in turn was often followed by a short cessation of activity before the recommencement of shorter duration bursting (Fig. $2 \mathrm{~A}$, middle). We did not analyze relative changes in those various discharge burst patterns. The further removal of rostral medulla in wild-type preparations caused no significant changes in the frequency (12.1 \pm 4.6 bursts/ min without rostral medulla) of respiratory rhythm. All three types of preparations isolated from $L b \times 1$ mutant mice generated much slower, yet regular, respiratory rhythms relative to corresponding wild-type preparations $(n=5)$. The removal of the pons and rostral medulla did not significantly affect the rhythmic discharge in mutant preparations. In addition, the longer duration, nonrespiratory rhythms were prominent in all three types of Lbx1 mutant preparations.

\section{Neuromodulation of respiratory rhythm in E18.5 Lbx1 mutant mice}

The slower respiratory rhythms generated by E18.5 Lbx1 mutant versus control could be attributable to an inability of the preBötC

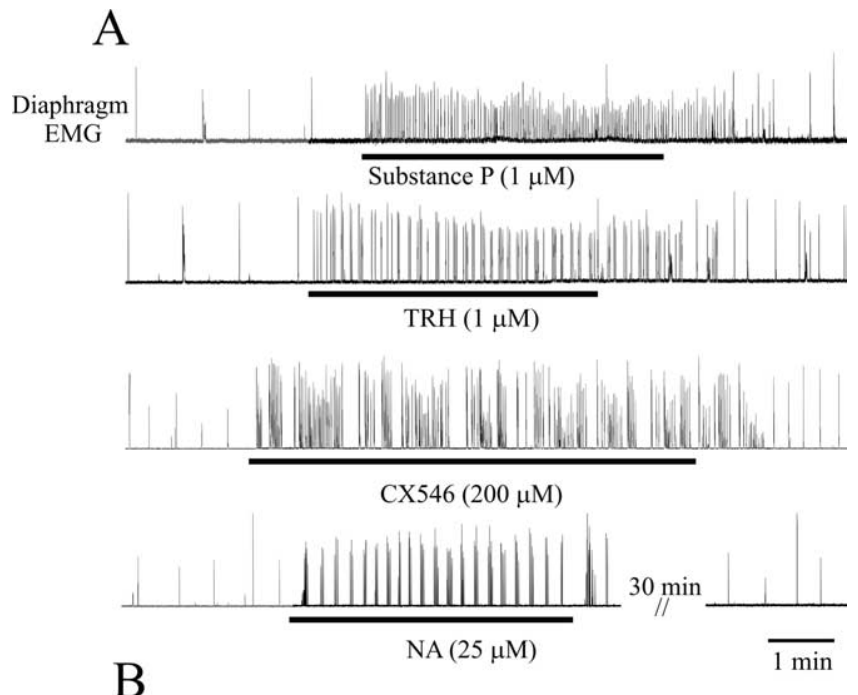

B

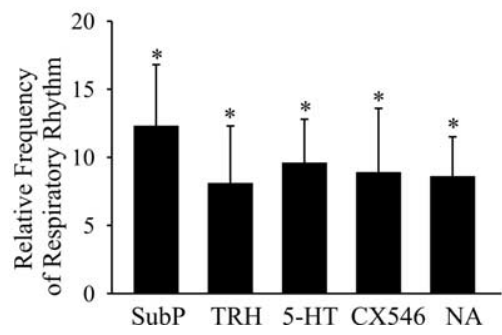

Figure 3. Effects of excitatory neuromodulators on respiratory rhythm generated by E18.5 Lbx1 mutant brainstem-spinal cord-diaphragm preparations. $A$, Examples of integrated, rectified diaphragm EMG recordings from the brainstem-spinal cord-diaphragm preparations of E18.5 Lbx1 mutant mice show excitatory actions of SubP 1( $\mu \mathrm{M})$, TRH (1 $\mu \mathrm{M})$, ampakine CX546 $(200 \mu \mathrm{M})$, and noradrenaline $(25 \mu \mathrm{M}) . \boldsymbol{B}$, Population data showing respiratory frequency relative to control after addition of each agent as well as $5-\mathrm{HT}(25 \mu \mathrm{M})$. Each data point was from four to five preparations tested. ${ }^{*} p<0.05$ relative to control.

rhythm generating network to generate faster rhythms. Alternatively, it may reflect defects in the neuromodulatory systems that provide conditioning excitatory/inhibitory drive to the preBötC or a lack of sufficient synaptic drive among neurons within the preBötC network. To determine whether the respiratory frequency could be increased, we bath applied neuromodulators known to provide excitatory drive to the preBötC. This included substance $\mathrm{P}$ (SubP; $1 \mu \mathrm{M}$ ), thyrotropin releasing hormone (TRH; $1 \mu \mathrm{M})$, serotonin (5-HT; $25 \mu \mathrm{M})$, or noradrenaline $(25 \mu \mathrm{M})$ (AlZubaidy et al., 1996; Greer et al., 1996; Viemari and Gérard, 2002; Pagliardini et al., 2003). We also applied the ampakine drug 1-(14-benzodioxan-6-yl-carbonyl) piperidine (CX546) (200 $\mu \mathrm{M})$, a positive modulator of AMPA receptors, that has been shown to increase respiratory frequency by increasing the efficacy of endogenous glutamatergic synaptic drive (Ren et al., 2006). Diaphragm EMG recordings from brainstem-spinal cord-diaphragm preparations were used to monitor respiratory rhythm. Figure 3 shows that all of the excitatory agents caused a very pronounced increase in the frequency of respiratory rhythm in E18.5 Lbx1 mutant preparations. These data demonstrate that Lbx1 mutant mice at E18.5 are capable of generating robust respiratory rhythms similar to wild-type mice when provided with the necessary excitatory conditioning neurochemical drive.

We also tested whether the slow rhythms were in part caused by increased inhibitory drive via GABAergic or glycinergic mechanisms (Cheng et al., 2004). There were no significant changes in respiratory frequency or variability after bath application of the 
receptor antagonists bicuculline (free base, $10 \mu \mathrm{M} ; n=3)$ or strychnine $(1 \mu \mathrm{M}$; $n=3)$. The frequency and coefficient of variability in control, bicuculline, and strychnine conditions were $1.4 \pm 0.56$ bursts/min and $0.7 \pm 0.16,1.57 \pm 0.9$ bursts/min and $0.68 \pm 0.21$, and $1.47 \pm$ 0.57 bursts $/ \mathrm{min}$ and $0.60 \pm 0.12$, respectively.

\section{Anatomical analyses of respiratory nuclei of wild-type and $L b x I$ mutant mice}

To investigate neuroanatomical abnormalities that may explain respiratory defects in $L b x 1$ mutant mice, we studied the expression of different neuronal markers within the caudal pons and medulla. For the purpose of this study, we primarily focused on the expression patterns and anatomical defects relevant to the key nuclei involved in modulating respiratory function and those data are discussed in detail below. However, for completion, we did determine Lbxl expression patterns and several defects in $L b x 1$ mutants within other brainstem regions. Figure 4 shows the expression patterns of GFP in the caudal and rostral medulla of $L b \times 1^{G F P /+}$ mice and some of the major defects in the mutant $L b x 1^{G F P / G F P}$ mice. In heterozygote mice, GFP is expressed by cells in the spinal trigeminal subnuclei [caudalis $(\mathrm{SpVc})$ and interpolaris $(\mathrm{SpVi})]$, in the cuneate and gracile nuclei $(\mathrm{Cn} / \mathrm{Gr})$, in the area postrema (AP), in the NTS, in scattered cells of the ventral medulla below the NA, in the parvicellular reticular formation (PR), in the gigantocellular reticular formation $(\mathrm{Gi})$, and in the vestibular nucleus (Ve).

The cytoarchitecture of the medulla of $L b \times 1^{G F P / G F P}$ mice was severely disrupted at E18.5. In particular, the sensory nuclei located in the dorsolateral medulla $(\mathrm{Cn} / \mathrm{Gr}$, $\mathrm{SpV}$, and NTS) were highly abnormal, as reported previously from Sieber et al. (2007). The SpV failed to form and neurons rather bundled around the NTS. In addition to the dorsal defects reported previously, we demonstrate a reduced distribution of $\mathrm{GFP}^{+}$cell in the ventral medulla (Fig. 4, arrowhead) and a marked enlargement of the inferior olive (Fig. 4, arrows; supplemental Fig. 1, available at www.jneurosci.org as supplemental material). Furthermore, in $L b \times 1^{G F P / G F P}$ mice (E18.5), ascending and descending tracts were misrouted, and extensive longitudinally oriented fibers along the surface of the ventral medulla shifted ventral motoneuronal pools [NA and facial (VII) nuclei] to a more dorsolateral position relative to wild-type mice (data not shown). However, analysis of size extension and cell density of NA, VII, and XII showed no apparent defects in the respiratory related motoneuronal pools.

\section{PreBötC}

Neurokinin 1 receptor (NK1R) and SST immunoreactivity in the ventrolateral medulla identifies the region of the preBötC (Gray et al., 1999; Guyenet et al., 2002; Pagliardini et al., 2003). The

\section{$L b \times 1^{G F P / G F P}$}
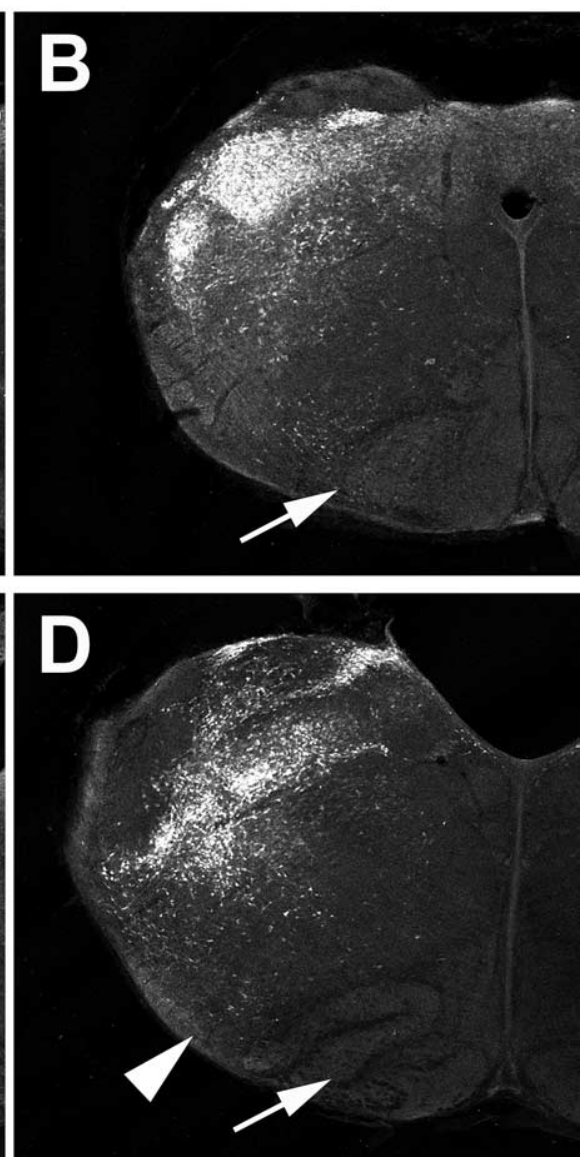

Figure 4. Lbx1 expression in wild-type and mutant brainstem. $\boldsymbol{A}-\boldsymbol{D}, \mathrm{GFP}$ expression in the caudal $(\boldsymbol{A}, \boldsymbol{B})$ and $\operatorname{rostral}(\boldsymbol{C}, \boldsymbol{D})$ medulla of $\angle b \times 1^{\text {GFP/+ }}$ and $L b \times 1^{\text {GFP/GFP }}$ mice atE18, respectively. GFP is expressed in the spinal trigeminal subnuclei (caudalis, SpV and interpolaris SpVi), in the (n and $\mathrm{Gr}$, in the AP, in the NTS, in scattered cells in the ventral medulla below the nucleus ambiguus, in the PR, and in the Ve in $L b \times 1^{G F P /+}$ mice. In $L b x 1^{G F P / G F P}$ mice, GFP is expressed in the putative region of SpV, $\mathrm{Cn}, \mathrm{Gr}, \mathrm{AP}$ where the cytoarchitecture of the sensory systems ( $\mathrm{SpV}, \mathrm{Cn}$ and $\mathrm{Gr}$ ) is grossly abnormal. In addition, there is a reduction of cells in the ventral medulla (arrowhead in D) and a marked enlargement of the inferior olive (arrows in $\boldsymbol{B}, \boldsymbol{D})$ ). Scale bars, $400 \mu \mathrm{m}$.

$\mathrm{NK}_{1 \mathrm{R}}{ }^{+}$and $\mathrm{SST}^{+}$cells in the preBötC area are not $L b x 1$ derived as they did not express GFP in $L b \times 1^{G F P /+}$ or $L b x 1^{\text {GFP/GFP }}$ mice. Furthermore, immunolabeling for NK1R and SST showed that the gross structure of the preBötC formed normally in mutant mice. However, in mutant mice preBötC location was shifted in a more dorsolateral position (Fig. 5), further away from the ventral surface of the medulla because of the aberrant migration of other medullary and spinal neurons, fiber tracts, and the expansion of the inferior olive.

\section{RTN neurons}

Data from recent studies suggests that a population of glutamatergic neurons ventral to the VII nucleus that express Phox $2 \mathrm{~b}$ directly sense the $\mathrm{pH}$ of the blood and provide a major chemosensory drive to the preBötC (Stornetta et al., 2006; Mulkey et al., 2007). This population corresponds to the classical location and phenotype described for chemosensitive neurons of the RTN (Feldman et al., 2003; Mulkey et al., 2004; Stornetta et al., 2006). Furthermore, mutant mice lacking Phox2b-positive neurons in the RTN die shortly after birth because of respiratory dysfunction (Dubreuil et al., 2008) similar to Lbx1 mutants. We found that the majority of Phox 2b-expressing RTN neurons in $L b \times 1^{G F P /+}$ mice were GFP positive and also expressed the NK1R (Fig. 6A,C). 


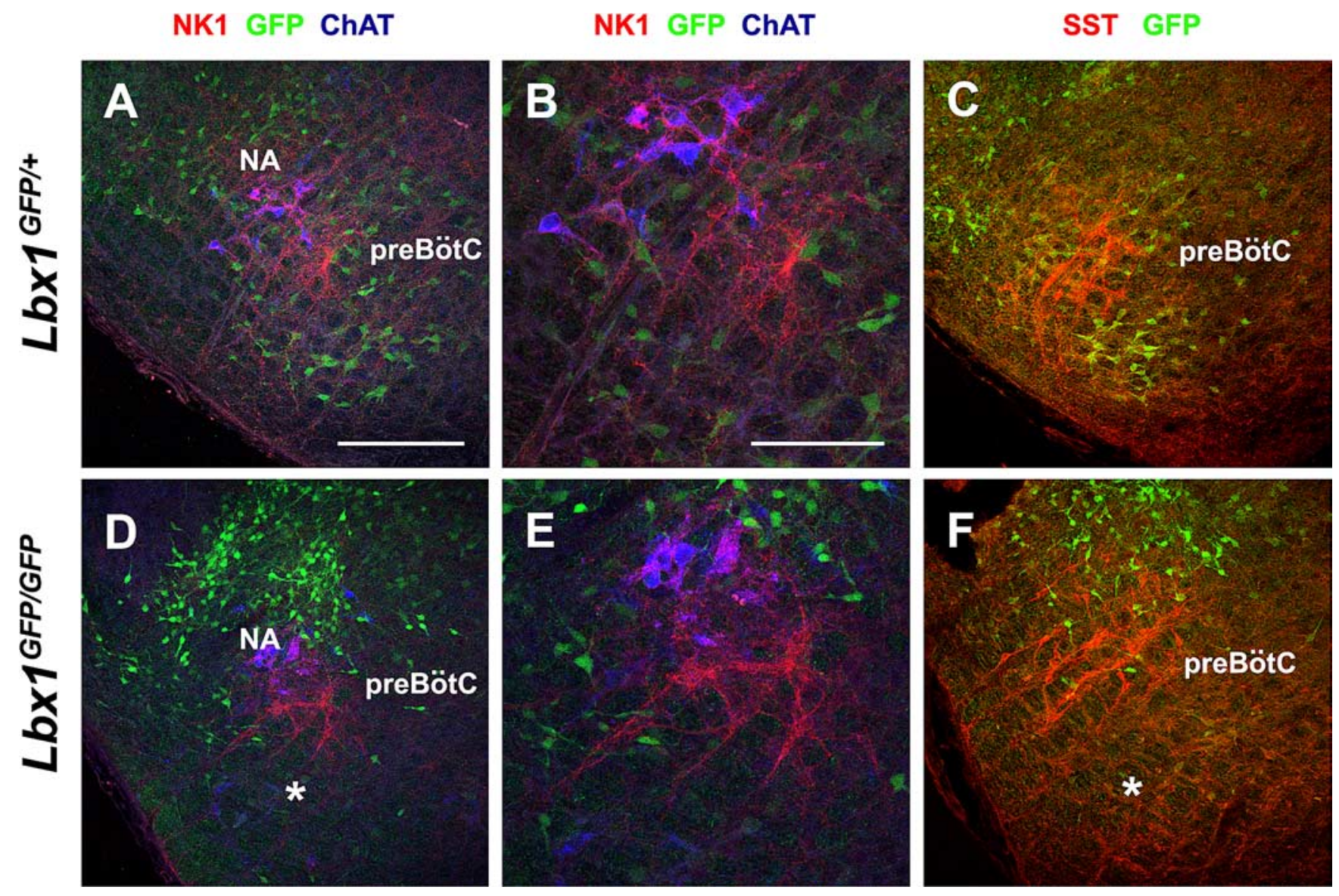

Figure 5. $A-F, G F P$ (green, $A-F)$, NK1R (red, $\boldsymbol{A}, \boldsymbol{B}, \boldsymbol{D}, \boldsymbol{E})$, ChAT (blue, $\boldsymbol{A}, \boldsymbol{B}, \boldsymbol{D}, \boldsymbol{E})$, and SST (red, $\boldsymbol{C}, \boldsymbol{F})$ expression in the ventrolateral medulla of $L b x 1^{G F P /+}$ and $L b x 1^{G F P / G F P}$ mice at $E 18$. PreBötC neurons (NK1R ${ }^{+} / \mathrm{ChAT}^{-}$and SST ${ }^{+}$) are present in both $L b x 1^{G F P /+}$ and $L b x 1^{G F P / G F P}$ mice. $B, E$, Details of preBötC neurons from $A$ and $\boldsymbol{D}$, respectively. There is a marked reduction in GFP ${ }^{+}$neurons within the network of the ventrolateral medulla (asterisks). Scale bars: $\boldsymbol{A}, \boldsymbol{C}, \boldsymbol{D}, \boldsymbol{F}, 200 \mu \mathrm{m} ; \boldsymbol{B}, \boldsymbol{E}, 100 \mu \mathrm{m}$.

Furthermore, Phox2b/NK1R-expressing neurons in the RTN were absent in $L b x 1$ mutant mice (Fig. $6 B, D$ ). Those data demonstrate that $L b \times 1$ is necessary for the proper development of a subclass of Phox2b/NK1R RTN neurons in the caudal pons.

\section{Catecholaminergic neurons}

Respiratory function is strongly modulated by catecholaminergic neurons in pontine and medullary structures (Li et al., 2008). Many of these catecholaminergic neurons are also specified by Phox $2 b$, however they originate from a distinct developmental population than glutamatergic RTN neurons (Pattyn et al., 2000; Qian et al., 2001; Brunet and Pattyn, 2002; Dauger et al., 2003) and they do not coexpress GFP in $L b \times 1^{G F P /+}$ mice (Fig. 7A-C). All of the catecholaminergic nuclei are present in $L b \times 1$ null mice (Fig. $7 D-F$ ). However, loss of $L b x 1$ activation did cause a perturbation within these populations, as the numbers of TH-positive neurons were increased in the catecholaminergic groups $\mathrm{A} 1 / \mathrm{C} 1$ $(158.1 \pm 15.9 \% ; n=6)$ and A5 $(222.8 \pm 63.7 \% ; n=3)$ nuclei in mutant mice (Fig. 7). A similar increase in TH-positive neurons was also observed dorsal to the nucleus ambiguus $(246.5 \% \pm 95.2$ $\mathrm{SD} ; n=5)$ and along the midline $(288.0 \pm 157.5 \% \mathrm{SD} ; n=4)$. Increased staining for TH fibers and neurons, were also observed in the $\mathrm{A} 2 / \mathrm{C} 2$ and $\mathrm{C} 2 / \mathrm{C} 3$ catecholaminergic group. Collectively, the data suggest that loss of Lbx1 expression results in ectopic noradrenergic neuron expression.

We tested the hypothesis that the excess TH-positive neuronal populations resulted in increase noradrenergic input to the preBötC and influenced rhythmogenesis in mutant brainstem-spi- nal cord preparations. Bath application of $\alpha 1$ and $\alpha 2$ adrenoceptor antagonists prazosin $(50 \mu \mathrm{M})$, and idazoxan $(50 \mu \mathrm{M})$ had no significant effects on the respiratory frequency in mutant $(n=3)$ and wild-type $(n=3)$ preparations (data not shown).

GABAergic and glycinergic inhibitory inputs

Studies of spinal cord development have shown that $L b \times 1$ is expressed in a subset of inhibitory neurons that express Pax2 (Burrill et al., 1997). Furthermore, mutant mouse models lacking substantial populations of GABAergic and glycinergic medullary neurons have a lethal respiratory phenotype (Kuwana et al., 2003; Fujii et al., 2007), consistent with what we observed with our electrophysiolgocial recordings of $L b \times 1$ mutants. Thus, we focused on examining inhibitory neurons within the NTS and the ventrolateral medulla, given that these regions are involved in modulating respiratory rhythm.

Figure $8 A-D$ shows the distribution of $L b \times 1$-derived, Pax2expressing inhibitory neurons in $L b x 1^{G F P /+}$ or $L b x 1^{G F P / G F P}$ mice. A widespread reduction of $\mathrm{Pax} 2 / \mathrm{GFP}$-coexpressing neurons was observed in the sensory structures of $\mathrm{Cn} / \mathrm{Gr}$, in the $\mathrm{SpV}$ and in neurons of the ventral medulla and NTS. In mutant mice, Pax $2^{+} /$ $\mathrm{GFP}^{-}$neurons were apparently not affected by the absence of $L b \times 1$ during development. Figure $8 E-H$ shows Pax 2 expression in the ventral medulla. Here, we observed in $L b x 1^{G F P /+}$ mice, two distinct Pax2-expressing populations, one that is Lbx1 derived. In $L b \times 1^{G F P / G F P}$ mice (Fig. $8 F, H$ ), the population of inhibitory $\mathrm{GFP}^{+} / \mathrm{Pax}^{+}$neurons is substantially decreased in this region.

The major inhibitory neurotransmitter in the respiratory col- 
umn is glycine (Shao and Feldman, 1997). The best characterized inhibitory population in the ventrolateral medulla is the glycinergic expiratory neurons of the BötC. Figure $9, A$ and $C$, shows that $L b \times 1$-derived neurons in the BötC express Pax2. Combined in situ hybridization for GlyT2(Slc6a5) (Fig. 9B,D) and immunohistochemistry for Pax2 (Fig. 9D) in the adjacent section demonstrates that BötC neurons are glycinergic and Lbxl derived. Contrasting GlyT2 mRNA labeling in wild-type (Fig. 9B) and mutant (Fig. 9E) mice demonstrates the necessity of $L b \times 1$ expression for development of glycinergic neurons in the ventral medulla. Furthermore, the loss of Lbx1 expression leads to severe migration abnormalities of GFP/Pax2expressing neurons (Fig. 9F).

Given the marked loss of glycinergic and GABAergic neurons in the medulla, we tested whether exogenous application of those neurotransmitters would normalize the slow rhythms in mutant brainstem-spinal cord preparations. However, bath application of the $\mathrm{GABA}_{\mathrm{A}}$ receptor agonist muscimol $(0.1 \mu \mathrm{M})$ or glycine (30 $\mu \mathrm{M})$ resulted in yet further slowing of the respiratory rhythm $(\sim 50 \% ; n=3$; data not shown).

\section{Origins of anatomical defects in respiratory nuclei}

The above data demonstrate that the absence of $L b x 1$ expression results in a marked reduction of Phox $2 b$ neuronal populations within the RTN and inhibitory neurons within multiple nuclei at E18.5. We examined the hindbrain of $L b x 1^{G F P /+}$ and $L b x 1^{G F P / G F P}$ mice at E10.5-E12.5 to determine whether these defects were present early in development or were caused by some later stage developmental abnormality. A recent study has classified dorsally generated neuronal populations in the brainstem based on the expression of specific transcription factors (Sieber et al., 2007). This organization is summarized in Figure $10 \mathrm{~A}$. Briefly, most dorsalgenerated neurons are classified into four subpopulations (dA1$\mathrm{dA} 4$ ), and more ventrally generated neurons are classified in four subpopulations $(\mathrm{dB} 1-\mathrm{dB} 4)$. Two additional subpopulations of neurons (dBLA-dBLB) are generated later in development. During the first wave of neurogenesis at E10.5-E11.5, Lbx1expressing cells are present in $\mathrm{dB} 1, \mathrm{~dB} 2$ (exclusively in the pons), $\mathrm{dB} 3$ and $\mathrm{dB} 4$ domains in the developing hindbrain. During the second wave of neurogenesis at E12.5, Lbx1 neurons develop in $\mathrm{dBL}_{\mathrm{A}}$ and $\mathrm{dBL}_{\mathrm{B}}$ populations (Sieber et al., 2007; Gray, 2008). We confirmed the classification of the Lbx1-expressing neurons outlined in Sieber et al., 2007 and further extended the analysis on the origin, identity and likely neurotransmitter phenotype of these neuronal populations in both wild-type and mutants.

\section{Inhibitory interneurons}

When we studied the expression of Pax 2 and Lmx1b in the early stages of development (Fig. $10 B-E$ ), we confirmed the absence of Pax2 from $\mathrm{dB} 1$ neurons as early as when the first $\mathrm{dB}$ neurons are generated (Fig. 10C,E) (data not shown). Furthermore, in late generated $\mathrm{dLB}_{\mathrm{A}}$ neurons, Pax2 expression is again absent (Fig. $10 G, I)$. These results suggest that $P a \times 2 / L b \times 1$ inhibitory neurons in the medulla do not form and putative neurons are respecified into a more dorsally generated FoxP2 (dA2 neurons of inferior olive) (supplemental Fig. 1, available at www.jneurosci.org as supplemental material) and Phox2b-dependent neurons (dA3, that later in development settle in the dorsal medulla) (Sieber et al., 2007). Thus, the loss of glycinergic Pax2 positive neurons in the ventral medulla at E18 could be accounted for abnormal specification during the E10-E12 period of neurogenesis.

\section{Phox2b RTN neurons}

Because $L b \times 1 /$ GFP is exclusively coexpressed with Phox $2 b$ in the dB2 population of $L b \times 1^{G F P /+}$ mice (Fig. 10, supplemental Fig. 2, available at www.jneurosci.org as supplemental material), we propose that RTN neurons originate solely from dB2 Lbx1/ Phox2b-expressing neurons, in contrast with other cathecholaminergic and cholinergicneurons in the brainstem, which originate from dA3 neurons. In the early stages of development of $L b \times 1^{G F P / G F P}$ mice we could not detect any abnormalities in the pontine origin of $\mathrm{dB} 2$ neurons (supplemental Fig. 2, available at www.jneurosci.org as supplemental material); therefore, it is likely that many of the putative Phox2b/Lbx1 RTN neurons originate from $\mathrm{dB} 2$ but fail to properly migrate to the ventral surface of the caudal pons. In addition, we observed a large increase in Phox $2 b$-expressing neurons within the $\mathrm{dB} 3$ and $\mathrm{dBl}$ populations of $L b \times 1^{G F P / G F P}$ mice medulla. Similar to what was reported by Sieber et al. (2007), we observed that the majority of the putative 

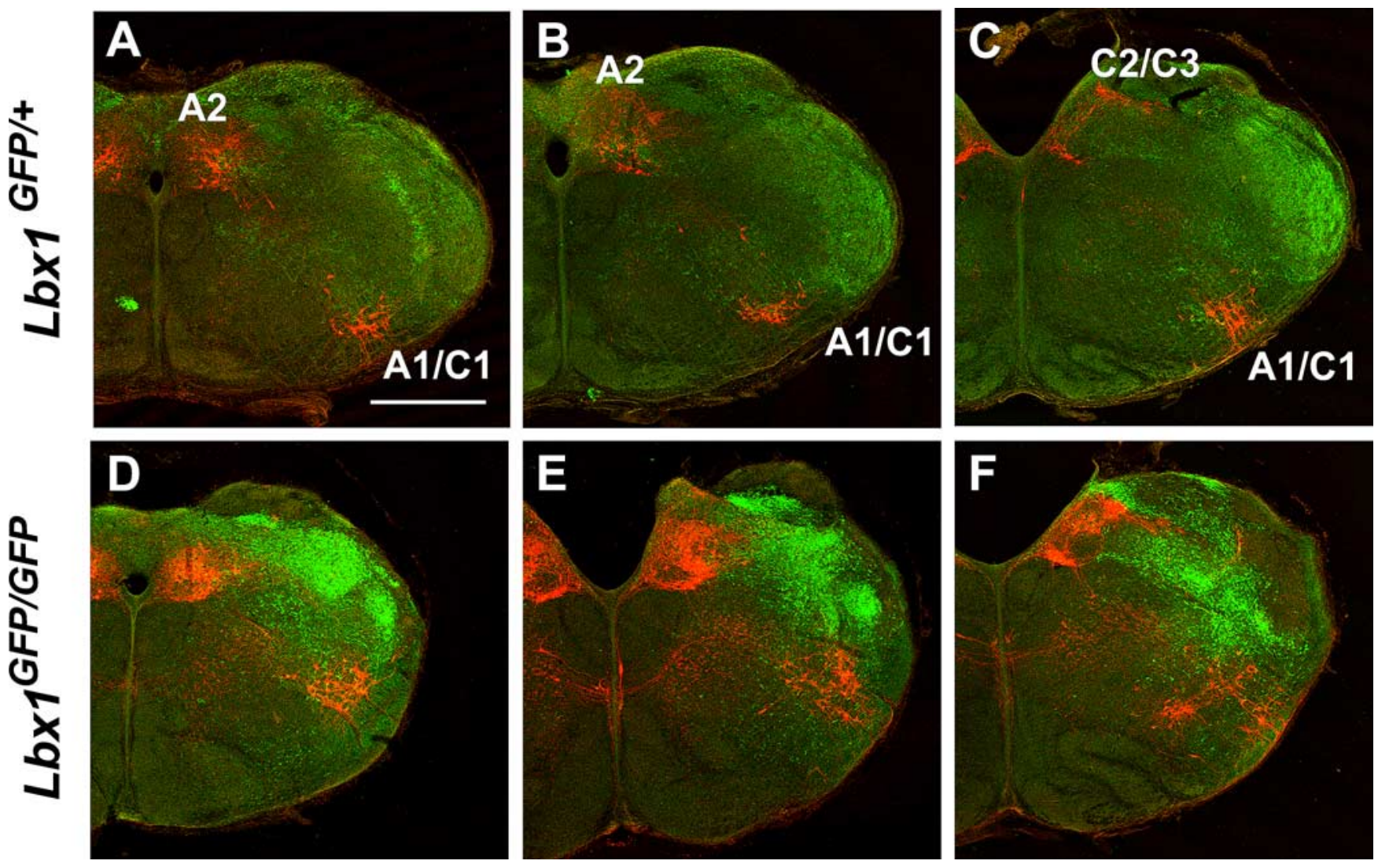

Figure 7. Ectopic expression of TH-positive neurons. $\boldsymbol{A}-\boldsymbol{F}$, Serial caudorostral transverse sections of an $\mathrm{E} 18$ medulla of $L b x]^{G F P /+}(\boldsymbol{A}-\boldsymbol{C})$ and $\left.L b x\right]^{G F P / G F P}(\boldsymbol{D}-\boldsymbol{F})$ mice labeled for TH (red) and GFP (green). Note the intense somatic and neuropilar staining in the noradrenergic structures of $A 1 / C 1, A 2, C 2 / C 3$ of $L b x 1^{G F P / G F P}$ mice. In addition, there is ectopic localization of $T H+$ neurons along the midline and in the reticular formation. Scale bar: $A-F, 500 \mu \mathrm{m}$.

Lbx1-derived neurons switched their neuronal phenotype and started expressing Phox $2 \mathrm{~b}$ and Lmx1b, typical markers of the more dorsally derived dA3 neurons that give origin to NTS, AP, and several cathecolaminergic populations (Fig 10). Therefore, the identification of the exact location of the misrouted RTN neurons was masked by the ectopic presence of Phox2b/GFPexpressing neurons in the brainstem of $L b x 1^{G F P / G F P}$ mice.

\section{Discussion}

Abnormal respiratory rhythmogenesis caused by loss of Lbx1 expression

In vitro recordings demonstrated that mice lacking Lbx1 expression have a markedly depressed frequency of respiratory rhythm during the late stages of gestation and at birth compared with wild-type mice. However, in vitro, the inception of respiratory rhythmogenesis in Lbxl mutants commenced at the same developmental stage as the wild-type mice. Indeed, at that early stage, there were no significant differences in respiratory frequency between wild-type and Lbx1 mutant preparations. By E18.5, the frequency of respiratory rhythm had increased approximately fivefold in the wild-type while not changing significantly from the E15.5 frequency in $L b x 1$ mutants. This raises the question of what is responsible for the typical increase in frequency of respiratory rhythm that occurs in vitro and in vivo during late fetal development. Potential mechanisms include: (1) age-dependent differences in neurons and/or the network underlying rhythmogenesis, (2) suppression of fetal network activity caused by endogenous inhibitory modulators, and (3) improper development of modulatory systems that provide excitatory drive to or within respiratory networks (Greer et al., 1992b; Kobayashi et al., 2001; Ren and Greer, 2003; Thoby-Brisson et al., 2005). In vitro, fetal rhythm-generating centers can oscillate at frequencies comparable to those of the neonate if agonists of excitatory modulators are administered (Greer et al., 2006). Similarly, in utero, the frequency of fetal breathing movements in rats is increased by administration of the respiratory stimulants doxapram and aminophylline (Kobayashi et al., 2001). Application of the excitatory neuromodulators TRH and SubP in vitro also produces markedly increases in the frequency of prenatal respiratory rhythm (Greer et al., 1996; Pagliardini et al., 2003). Here in this study, bath application of SubP, noradrenaline, 5-HT, and TRH revealed that the Lbx1 mutant E18.5 preparations could generate robust respiratory drive at frequencies similar to the wild-type if excitatory conditioning drive is added. Thus, the hypoventilation in Lbxl-deficient mice could be accounted for by a failure of the normal excitatory conditioning drive necessary to develop after E15.5.

Abnormal anatomical structures within pontomedullary structures that modulate respiratory rhythmogenesis in Lbx1 mutant mice.

There were no gross abnormalities of preBötC structure as defined by NK1R and SST labeling. The preBötC in $L b x 1$ mutants was positioned more dorsolaterally as a result of abnormal medullary nuclei and tract formation. This could result in perturbations in synaptic organization impinging onto the preBötC. However, the more substantial anatomical perturbations were with medullary and pontine structures that provide key modulatory drive to the preBötC. This included the following. 


\section{$L b \times 1^{G F P /+} L b \times 1^{G F P / G F P}$}
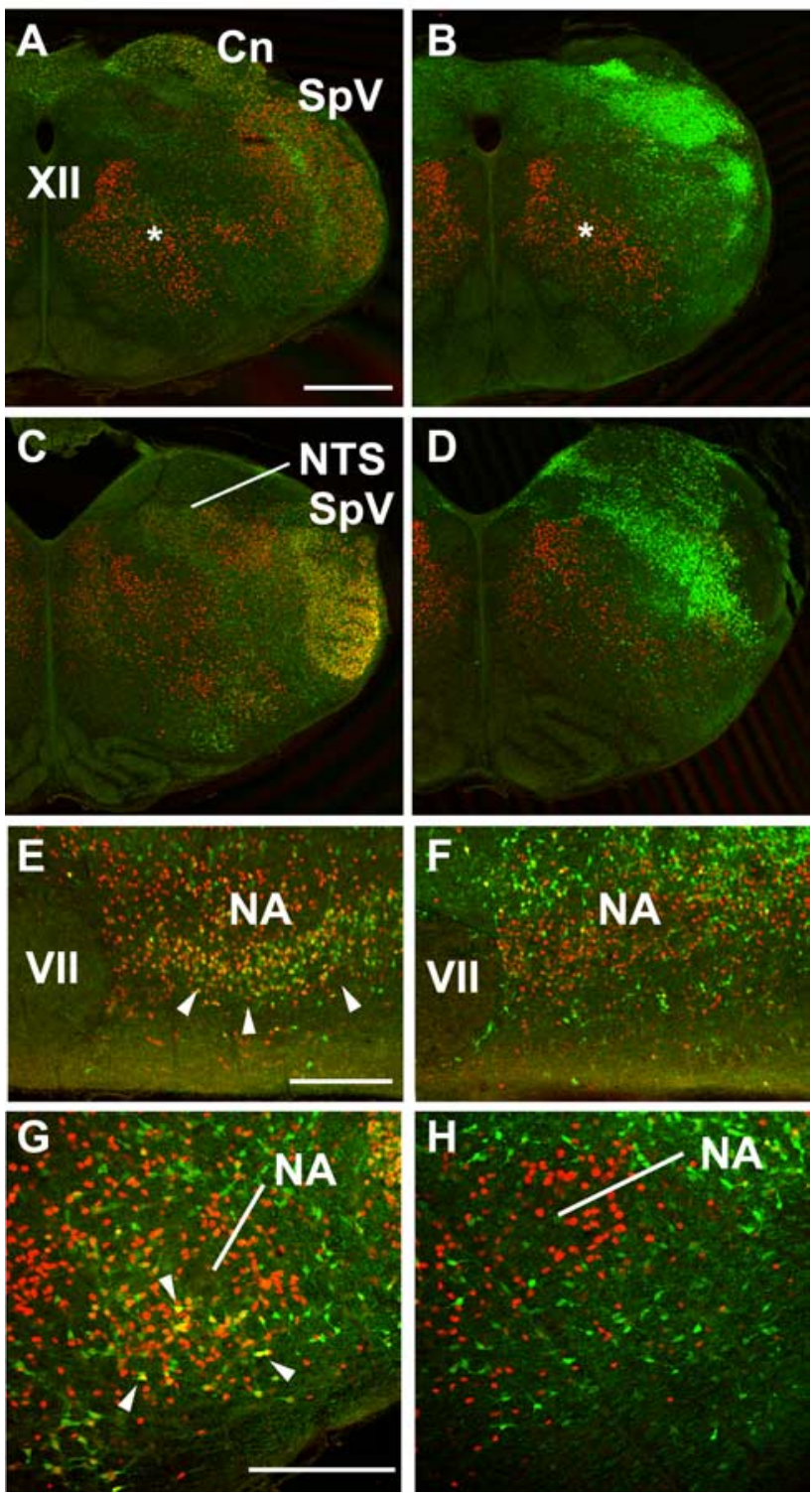

Figure 8. Pax2 (red) and GFP (green) expression in the medulla of $L b \times 1^{\text {GFP/+ }}$ and $L b \times 1^{\text {GFP/GFP }}$ mice at E18. $\boldsymbol{A}-\boldsymbol{D}$, Transverse section of caudal $(\boldsymbol{A}, \boldsymbol{B})$ and rostral medulla $(\boldsymbol{C}, \boldsymbol{D})$ labeled for Pax2 and GFP in $\angle b \times 1^{G F P /+}(\boldsymbol{A}, \boldsymbol{C})$ and $\angle b \times 1^{G F P / G F P}(\boldsymbol{B}, \boldsymbol{D})$ mice. In $\angle b \times 1^{G F P /+}$ mice, Pax2 is expressed in cells located in reticular formation and lateral to the XII nucleus (asterisk, $A$ ), in the $\mathrm{SpV}, \mathrm{Gr} / \mathrm{Cn}$, NTS nuclei and in the ventral medulla. Pax2 colocalizes with GFP in cells of $\mathrm{Cn} / \mathrm{Gr}$, SpV, and in a subpopulation of Pax $2^{+}$cells in the NTS and in the ventral medulla. $\ln \angle b \times 1^{\text {GFP/GFP }}$ mice Pax 2 is expressed in the medial reticular formation and lateral to XII nucleus (asterisks, $\boldsymbol{B}$ ), only scattered cells are present in the $\mathrm{Gr} / \mathrm{Cn}, \mathrm{SpV}$, NTS nuclei and in the ventral medulla. $\boldsymbol{E}-\boldsymbol{H}$, Parasagittal $(\boldsymbol{E}, \boldsymbol{F})$ and transverse $(\boldsymbol{G}, \boldsymbol{H})$ section details of the ventral medulla immunostained for Pax2 and GFP show the reduced number of neurons coexpressing Pax2 and GFP in the ventral medulla, below the NA, of $L b \times 1^{\text {GFP/GFP }}$ mice compared with $L b \times 1^{G F P /+}$ mice (arrowheads, $\boldsymbol{F}, \boldsymbol{G}$ ). Pax2 cells that do not express GFP are not affected in $L b \times 1^{G F P / G F P}$ mice. Scale bars: $A-D, 500 \mu \mathrm{m}$; $\boldsymbol{E}, \boldsymbol{F}, 200 \mu \mathrm{m} ; \boldsymbol{G}, \boldsymbol{H}, 100 \mu \mathrm{m}$.

Significant loss of glutamatergic RTN neurons in the region of the facial nucleus

Our data suggest that Phox2b/Lbx1 RTN neurons likely originate from the dB2 pontine population in both wild-type and mutants but they fail to properly migrate in $L b \times 1$ mutants. RTN neurons provide a major component of central chemosensitive drive to the preBötC. Thus, $L b x 1$ mutant mice lack a key element of tonic
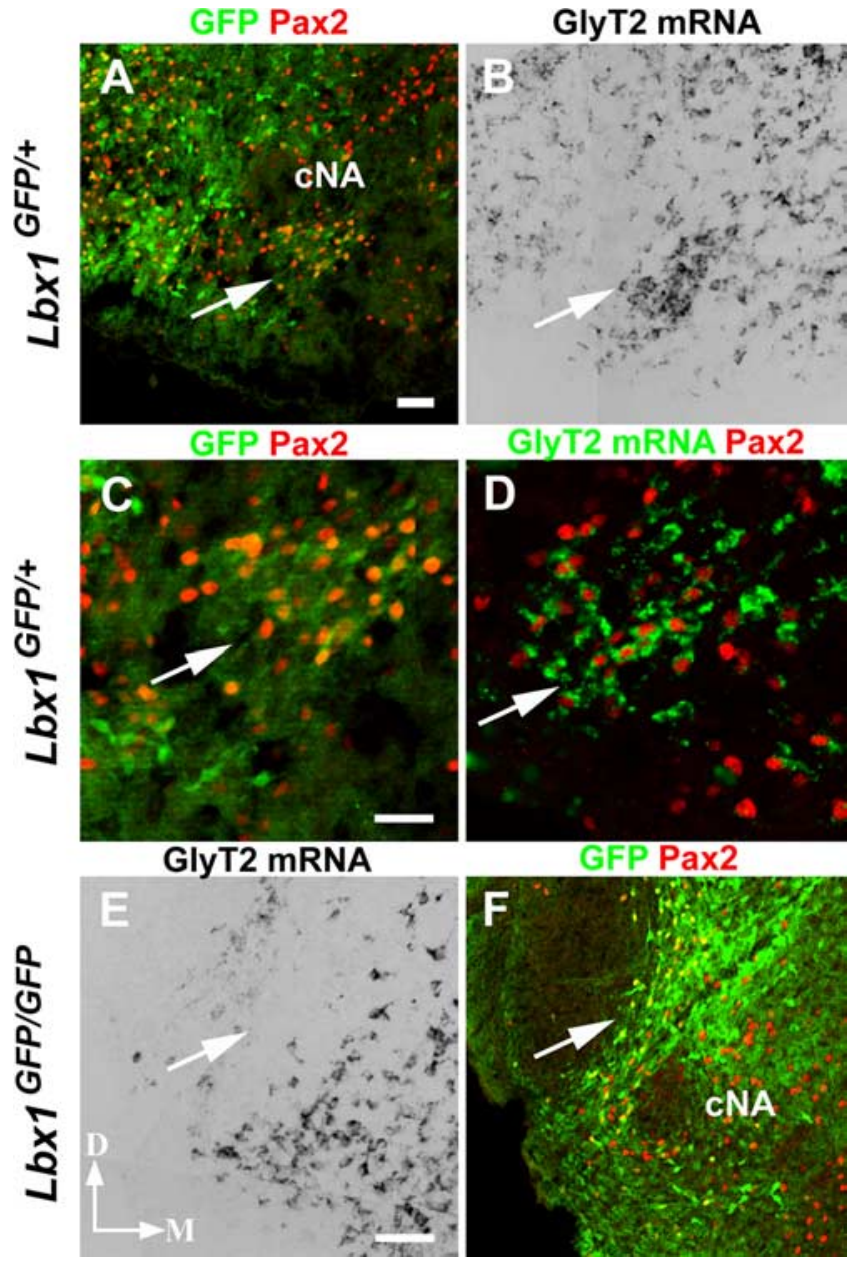

Figure 9. BötC glycinergic neurons are Lbx1 derived. $A$, Lbx1-derived GFP ${ }^{+}$(green) neurons in the BötC (arrow) express Pax2 (red). $\boldsymbol{B}$, In situ hybridization for GlyT2 in the adjacent Lbx ${ }^{\text {GFP/+ }}$ section identifies BötC neurons. C, Expanded image from $A$ showing GFP coexpression with Pax2 in the BötC. D, Pseudocolor image of coexpression of Pax2 protein (red) with GlyT2 mRNA (green) in $L b x 1^{G F P /+}$ mice section adjacent to $\boldsymbol{A}$. $\boldsymbol{E}$, In situ labeling for GlyT2 in $L b x 1^{G P P / G F P}$ mice BötC. $F$, Lbx1-derived neurons show a migration deficit in $L b x 1^{G F P / G F P}$ mice BötC. Scale bar: A, B, $200 \mu \mathrm{m} ; C, D, 200 \mu \mathrm{m} ; \boldsymbol{E}, F, 20 \mu \mathrm{m}$. D, Dorsal; M, medial.

excitatory synaptic drive and reflex compensatory drive in response to hypoxia resulting from apneas. Replacement of excitatory drive with bath application of excitatory neuromodulators or enhancement of remaining glutamatergic drive with ampakines normalized the respiratory rhythm in $L b x 1$ mutants. Consistent with our data, another mutant mouse model lacking Phox $2 b$ derived glutamatergic neurons in the RTN region die of central apnea at birth because of abnormal respiratory rhythms (Dubreuil et al., 2008).

Reduction of GABA and glycinergic input from the dorsolateral medulla, NTS, and ventrolateral medulla attributable to failure of the initial formation of $G A B A / g l y c i n e r g i c$ precursors with $\mathrm{dB1} /$ $d B L_{A}$ populations at $E 10$

There is typically very little tonic release of GABA or glycine that modulates respiratory rhythm in vitro (Zhang et al., 2002; Ren and Greer, 2006). One would not predict that a perturbation of those systems to significantly alter rhythmogenesis in vitro via alterations in membrane potential of preBötC neurons. Indeed, bath application of agonists to glycine and GABA receptors at E18 slowed the rhythm further and the addition of receptor antagonists did not alter rhythm. However, chloride-mediated conduc- 
Lbx $1^{\text {GFP/+ }}$

Atoh1, Lhx2/9

A

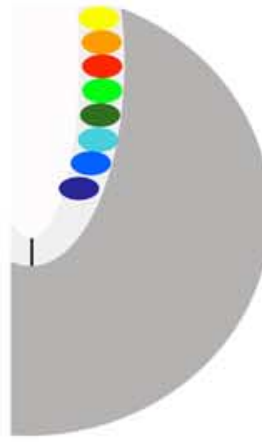

dA2 - Ptf1a,Lhx1/5, FoxP2

dA3 - Phox2b,Lmx1b,TIx3

dA4 - Lhx1/5

dB1 - Lbx1, Pax2, Lhx1/5

dB2 - Lbx1, Phox2b

dB3 - Lbx1, Lmx1b, TIx3

dB4 - Lbx1, Pax2, Lhx1/5

dBIA - Lbx1, Pax2, Lhx1/5

dBIB - Lbx1, Lmx1b, TIx3

\section{LbX1 GFP/GFP}

Atoh1, Lhx2/9

dA2 - Ptf1a,Lhx1/5, FoxP2

dA3 - Phox2b,Lmx1b,TIx3

dA4 - Lhx1/5

dB1 - Lbx1, Pax2, Lhx1/5, FoxP2

dB2 - Lbx1, Phox2b

dB3 - Lbx1, Lmx1b, TIx3, Phox2b

dB4 - Lbx1, Pax2, Lhx1/5

dBIA - Lbx1, Pax2, Lhx1/5

dBIB - Lbx1, Lmx1b, TIx3 , Phox2b
LbX1 GFP/+

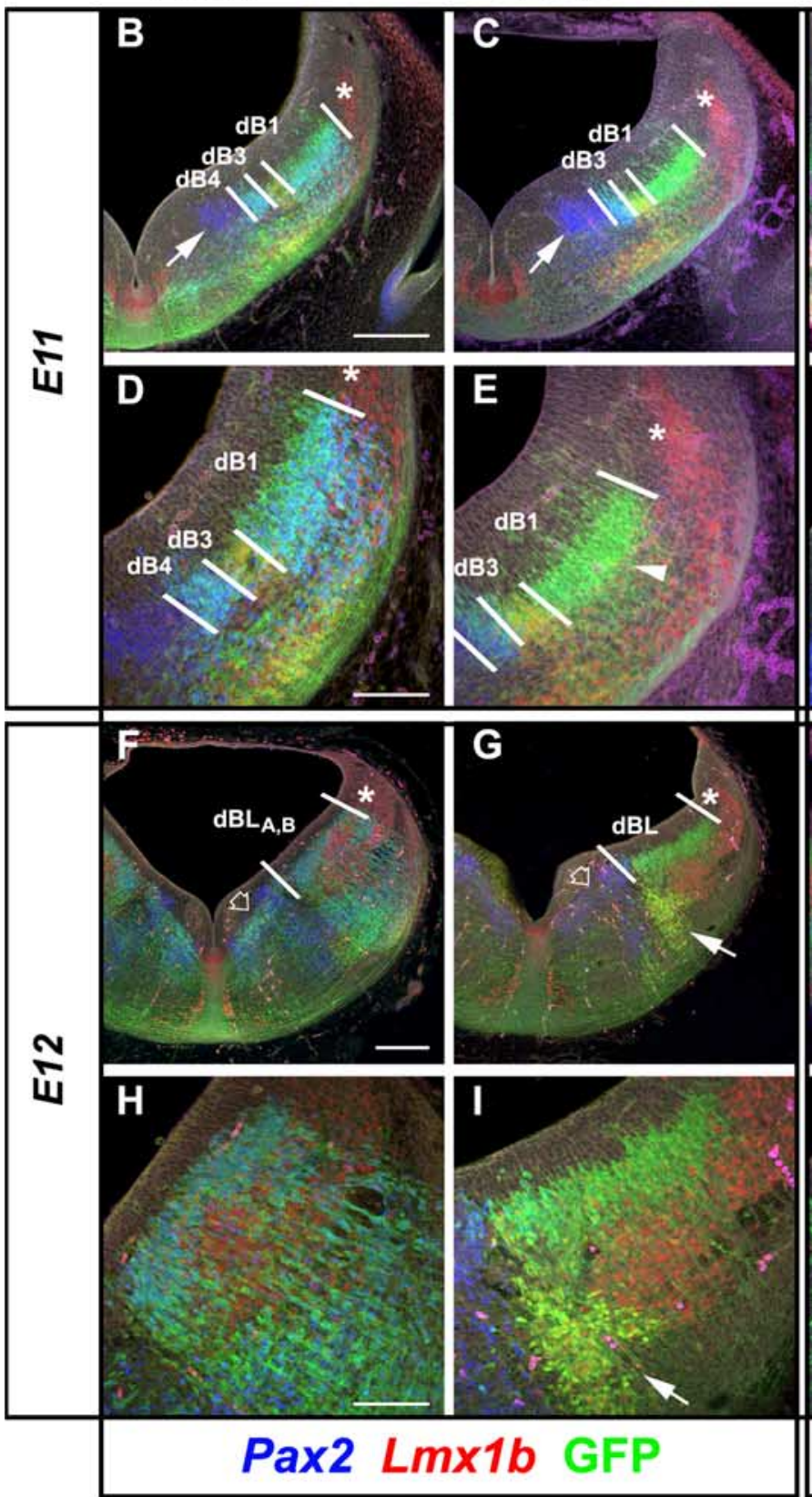

$L b \times 1^{G F P /+}$

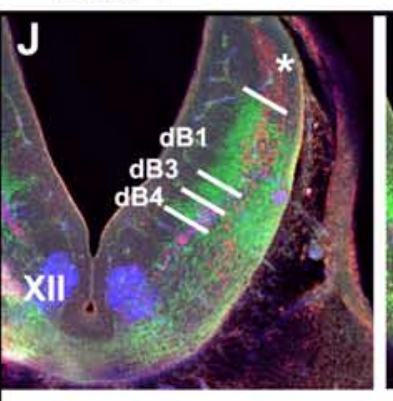

$\mathbf{L}$
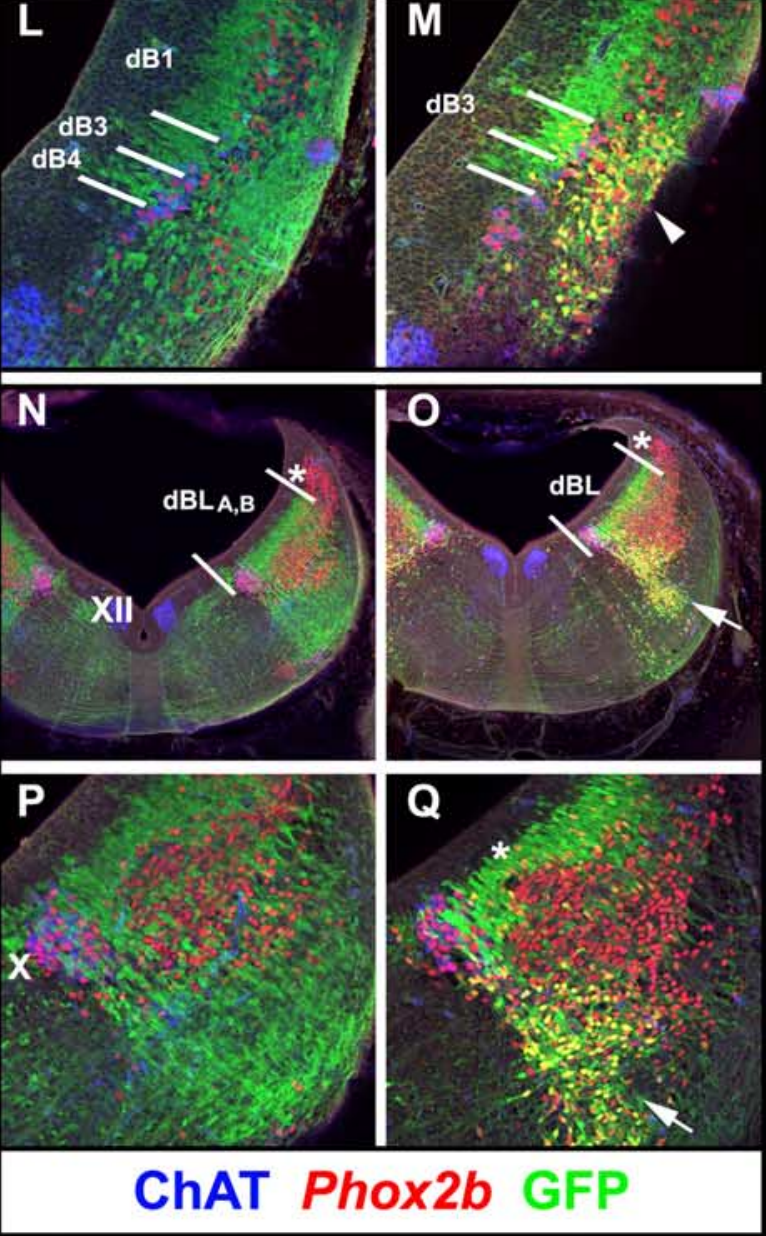
tances serve important roles in neuronal and network systems development. Specifically, at earlier stages (i.e., before E17) GABA and glycine act as excitatory neurotransmitters and promote growth factor expression and release (Gao and van den Pol, 2000; Ben-Ari, 2001; Gao and van den Pol, 2001; Gao et al., 2001). The loss of those actions may contribute to respiratory network and medullary structure abnormalities. Newborn mutant mice with deficiencies in GABA and glycine transmission have severely disrupted respiratory rhythm in vitro and in vivo (Kuwana et al., 2003; Fujii et al., 2007), similar to those generated by Lbx1 preparations lacking normal chloride-mediated neuromodulation during development.

\section{Ctopic catecholaminergic neuron formation caused by alterations} in neuronal phenotype

Catecholaminergic neurons are not essential for rhythmogenesis but they do modulate respiratory network function at multiple levels (for review, see Li et al., 2008). Noradrenaline induces an increase or decrease in respiratory frequency depending on the overall balance of $\alpha 1$ versus $\alpha 2$ receptor activation within the preBötC (Hilaire et al., 2004). We demonstrated that the abnormal respiratory rhythm generated in vitro did not result directly from abnormal activation of adrenergic receptors.

\section{Persistence of nonrespiratory rhythms because of loss of}

Lbx1 expression

Wild-type and Lbx1 mutant brainstem spinal cord preparations at E15.5 generate robust rhythmic bursting that are large in amplitude, longer in duration and more diffuse in its spread within the neuraxis relative to respiratory rhythms. Episodes of spontaneous rhythmic activity are widespread in the developing vertebrate nervous system (Katz and Shatz, 1996; Milner and Landmesser, 1999; Nakayama et al., 1999; O’Donovan, 1999; Hanson and Landmesser, 2003; Ren and Greer, 2003; Yvert et al., 2004). However, they typically subside post E15 in the developing mouse (Thoby-Brisson et al., 2005). The nonrespiratory rhythms are dependent on glutamatergic synaptic drive, suppressed by gap junction blockers, and originate in the spinal cord and dorsal regions of the medulla (Ren and Greer, 2003; Thoby-Brisson et al., 2005; Ren et al., 2006). Functionally, the persistence of the rhythms in Lbx1 mutant E18.5 in vitro preparations will result in

\section{$\leftarrow$}

Figure 10. Developmental origins of anatomical defects in respiratory neuronal populations. $\boldsymbol{A}$, Schematic expression of transcription factors in dorsally generated neurons of the hindbrain at $E 10$-E12 of $L b x]^{G F P /+}$ and $L b x 1^{G F P / G F P}$ mice: data are summarized from this paper, Sieber et al. (2007) and Gray (2008). Transcription factor in gray are not expressed in $\angle b x 7^{G F P / G F P}$ mice. Transcription factor ectopically expressed in mutants are indicated as purple. $\boldsymbol{B}-\boldsymbol{I}$, Expression of GFP, Pax2 and Lmx1b in E11 and E12 in $L b x 1^{G F P /+}$ and $L b x 1^{G F P / G F P}$ mice. In $L b x 1^{G F P /+}$ mice at E11, GFP (green) is expressed in the $\mathrm{dB} 1, \mathrm{~dB} 3, \mathrm{~dB} 4$ dorsal interneurons. $\operatorname{Lmx} 1 \mathrm{~b}$ (red) is expressed in the $\mathrm{dA} 3$ (asterisks) and $\mathrm{dB} 3$ dorsal interneurons, whereas Pax2 (blue) is expressed in the $\mathrm{dB} 1, \mathrm{~dB} 4$, and in ventral interneuronal populations (arrows in $\boldsymbol{D}, \boldsymbol{E}$ ). In $L b \times 1^{G F P / G F P}$ mice, Pax2 is absent in $\mathrm{dB} 1(\boldsymbol{E}$, arrowheads), whereas $\mathrm{Lmx} 1 \mathrm{~b}$ is expressed in $\mathrm{dA} 3$ and $d B 3$ dorsal interneurons. At E12, GFP is expressed in $\mathrm{dBL}_{\mathrm{A}-\mathrm{B}}$ dorsal interneurons of both $L b x 7^{G F P /+}$ and $L b x ~^{G F P / G F P}$ mice. $L m x 1 b$ is expressed in several interneurons $\left(\mathrm{dB}_{\mathrm{B}}\right.$ interneurons, $\left.G, I\right)$, whereas $\mathrm{Pax2}^{+} / \mathrm{GFP}^{+}$cells are missing in the $\mathrm{dBL}_{\mathrm{A}}$ neurons of $\angle b \times 1^{G F P / G F P}$ mice. Also note the absence of $\mathrm{Pax2}^{+} / \mathrm{GFP}^{+}$neurons in the medial medulla (open arrows in $\boldsymbol{F}, \boldsymbol{G}$ ) and the extensive GFP ${ }^{+} /$ $\mathrm{Lmx}_{\mathrm{bb}} \mathrm{b}^{+}$colocalization in the ventral medulla (arrows in $\mathbf{G}, I$ ). J, $\mathbf{Q}, \operatorname{In} L \mathrm{Lbx} 7^{\mathrm{GFP} /+}$ mice at E11, Phox $2 b$ (red) is expressed solely in $\mathrm{dA} 3$ neurons and in a subset of $\mathrm{ChAT}^{+}(J, L$, blue) motoneurons. In $L b x]^{G F P / G F P}$ mice, ectopic expression of Phox $2 b$ is mainly present in $\mathrm{dB} 3$ neurons (arrowhead in $M$ ). At E12, in $L b x 1^{G F P /+}$ mice, Phox $2 b^{+}$cells of $\mathrm{dA} 3$ origin are intermingled with Lbx1/GFP-expressing neurons, and no colocalization with GFP is apparent. In Lbx $1^{\text {GFP/GFP }}$ mice, extensive ectopic expression of Phox $2 \mathrm{~b}$ is present, both in early generated $\mathrm{dB} 3$ neurons (arrows in $\mathbf{O}, \mathbf{Q}$ ) and in late generated dBL neurons (asterisk in $\mathbf{Q}$ ). Scale bars: $\boldsymbol{B}, \boldsymbol{C}, \boldsymbol{J}, \boldsymbol{K}, 200 \mu \mathrm{m} ; \boldsymbol{D}, \boldsymbol{E}, \boldsymbol{L}$, $M, 100 \mu \mathrm{m} ; \boldsymbol{F}, \mathbf{G}, \mathbf{N}, \mathbf{O}, 200 \mu \mathrm{m} ; \boldsymbol{H}, \mathbf{I}, \mathbf{P}, \mathbf{Q}, 100 \mu \mathrm{m}$. an additional perturbation and occlusion of respiratory rhythm (Ren and Greer, 2003; Thoby-Brisson et al., 2005). The marked deficiency in the development of dorsal medullary inhibitory neurons in $L b x 1$ mutant mice likely contributes to the persistence of nonrespiratory rhythms.

\section{Functional implications for respiratory control}

Lbx1 mutant mice die at birth because of defective respiratory rhythmogenesis. Specifically, the respiratory frequency is slow and robust nonrespiratory rhythms persist throughout gestation that would interfere with coordinated respiratory motor patterns. Pharmacological experiments demonstrated that respiratory rhythm could be normalized with additional excitatory neurochemical drive to the preBötC. The loss of excitatory drive from neurons in the RTN was likely a major contributor to abnormal rhythmogenesis. Furthermore, loss of chloride-mediated synaptic input from the NTS, BötC, and ventrolateral medulla could lead to developmental abnormalities of neuronal function and network development. Overall, these data demonstrate that a malformation of conditioning drive from these structures has profound implications for normal breathing and survival. Mouse models of Rett, Prader Willi and Congenital Central Hypoventilation Syndromes all have central respiratory drive dysfunction caused by specific genetic defects (for review, see Gaultier, 2004; Pagliardini et al., 2008). In each case, data from those studies are consistent with the respiratory phenotype arising from multiple perturbations of conditioning drive to the preBötC rather than a defect of the preBötC per se. Furthermore, the cause of respiratory depression associated with apnea of prematurity, obstructive sleep apnea and potentially a subset of Sudden Infant Death have been hypothesized to result from abnormalities of conditioning synaptic inputs necessary for adequate respiratory rhythmogenesis and transmission (for review, see Darnall et al., 2006; Horner and Bradley, 2006). Thus, an understanding of the transcriptional control of not only the preBötc, but also the associated respiratory nuclei will be necessary for understanding the ontogeny of respiratory neural control and pathologies. Results from this study demonstrate that $L b \times 1$ is one of the critical "master genes" necessary for transcriptional control of key brainstem respiratory neural circuitry and function.

\section{References}

Al-Zubaidy ZA, Erickson RL, Greer JJ (1996) Serotonergic and noradrenergic effects on respiratory neural discharge in the medullary slice preparation of neonatal rats. Pflugers Arch 431:942-949.

Ben-Ari Y (2001) Developing networks play a similar melody. Trends Neurosci 24:353-360.

Burrill JD, Moran L, Goulding MD, Saueressig H (1997) PAX2 is expressed in multiple spinal cord interneurons, including a population of EN1+ interneurons that require PAX6 for their development. Development 124:4493-4503.

Cheng L, Arata A, Mizuguchi R, Qian Y, Karunaratne A, Gray PA, Arata S, Shirasawa S, Bouchard M, Luo P, Chen CL, Busslinger M, Goulding M, Onimaru H, Ma Q (2004) Tlx3 and Tlx1 are post-mitotic selector genes determining glutamatergic over GABAergic cell fates. Nat Neurosci 7:510-517.

Cheng L, Samad OA, Xu Y, Mizuguchi R, Luo P, Shirasawa S, Goulding M, Ma Q (2005) Lbxl and Tlx3 are opposing switches in determining GABAergic versus glutamatergic transmitter phenotypes. Nat Neurosci 8:1510-1515.

Dietrich S, Schubert FR, Healy C, Sharpe PT, Lumsden A (1998) Specification of the hypaxial musculature. Development 125:2235-2249.

Dutschmann M, Mörschel M, Kron M, Herbert H (2004) Development of adaptive behaviour of the respiratory network: implications for the pontine Kolliker-Fuse nucleus. Respir Physiol Neurobiol 143:155-165.

Feldman JL, Mitchell GS, Nattie EE (2003) Breathing: rhythmicity, plasticity, chemosensitivity. Annu Rev Neurosci 26:239-266. 
Fujii M, Arata A, Kanbara-Kume N, Saito K, Yanagawa Y, Obata K (2007) Respiratory activity in brainstem of fetal mice lacking glutamate decarboxylase 65/67 and vesicular GABA transporter. Neuroscience 146:1044-1052.

Gao XB, van den Pol AN (2000) GABA release from mouse axonal growth cones. J Physiol 523:629-637.

Gao XB, van den Pol AN (2001) GABA, not glutamate, a primary transmitter driving action potentials in developing hypothalamic neurons. J Neurophysiol 85:425-434.

Gao XB, Stricker C, Ziskind-Conhaim L (2001) Transition from GABAergic to glycinergic synaptic transmission in newly formed spinal networks. J Neurophysiol 86:492-502.

Gong S, Zheng C, Doughty ML, Losos K, Didkovsky N, Schambra UB, Nowak NJ, Joyner A, Leblanc G, Hatten ME, Heintz N (2003) A gene expression atlas of the central nervous system based on bacterial artificial chromosomes. Nature 425:917-925.

Gray PA, Rekling JC, Bocchiaro CM, Feldman JL (1999) Modulation of respiratory frequency by peptidergic input to rhythmogenic neurons in the preBötzinger complex. Science 286:1566-1568.

Gray PA (2008) Transcription factors and the genetic organization of brainstem respiratory neurons. J Appl Physiol 104:1513-1521.

Gray PA, Janczewski WA, Mellen N, McCrimmon DR, Feldman JL (2001) Normal breathing requires preBötzinger complex neurokinin-1 receptorexpressing neurons. Nature Neurosci 4:927-930.

Greer JJ, Smith JC, Feldman JL (1992a) Respiratory and locomotor patterns generated in the fetal rat brain stem-spinal cord in vitro. J Neurophysiol 67:996-999.

Greer JJ, Smith JC, Feldman JL (1992b) Glutamate release and presynaptic action of AP4 during inspiratory drive to phrenic motoneurons. Brain Res 576:355-357.

Greer JJ, al-Zubaidy Z, Carter JE (1996) Thyrotropin-releasing hormone stimulates perinatal rat respiration in vitro. Am J Physiol 271:R1160-1164.

Gross MK, Dottori M, Goulding M (2002) Lbx1 specifies somatosensory association interneurons in the dorsal spinal cord. Neuron 34:535-549.

Guyenet PG, Sevigny CP, Weston MC, Stornetta RL (2002) Neurokinin-1 receptor-expressing cells of the ventral respiratory group are functionally heterogeneous and predominantly glutamatergic. J Neurosci 22:3806-3816.

Hanson MG, Landmesser LT (2003) Characterization of the circuits that generate spontaneous episodes of activity in the early embryonic mouse spinal cord. J Neurosci 23:587-600.

Jagla K, Dolle P, Mattei MG, Jagla T, Schuhbaur B, Dretzen G, Bellard F, Bellard M (1995) Mouse Lbxl and human LBX1 define a novel mammalian homeobox gene family related to the Drosophila lady bird genes. Mech Dev 53:345-356.

Katz LC, Shatz CJ (1996) Synaptic activity and the construction of cortical circuits. Science 274:1133-1138.

Kobayashi K, Lemke RP, Greer JJ (2001) Ultrasound measurements of fetal breathing movements in the rat. J Appl Physiol 91:316-320.

Kuwana S, Okada Y, Sugawara Y, Tsunekawa N, Obata K (2003) Disturbance of neural respiratory control in neonatal mice lacking GABA synthesizing enzyme $67-\mathrm{kDa}$ isoform of glutamic acid decarboxylase. Neuroscience 120:861-870.

Mulkey DK, Stornetta RL, Weston MC, Simmons JR, Parker A, Bayliss DA,
Guyenet PG (2004) Respiratory control by ventral surface chemoreceptor neurons in rats. Nat Neurosci 7:1360-1369.

Müller T, Brohmann H, Pierani A, Heppenstall PA, Lewin GR, Jessell TM, Birchmeier C (2002) The homeodomain factor lbxl distinguishes two major programs of neuronal differentiation in the dorsal spinal cord. Neuron 34:551-562.

Nakayama K, Nishimaru H, Iizuka M, Ozaki S, Kudo N (1999) Rostrocaudal progression in the development of periodic spontaneous activity in fetal rat spinal motor circuits in vitro. J Neurophysiol 81:2592-2595.

O'Donovan MJ (1999) The origin of spontaneous activity in developing networks of the vertebrate nervous system. Curr Opin Neurobiol 9:94-104.

Oliva AA Jr, Jiang M, Lam T, Smith KL, Swann JW (2000) Novel hippocampal interneuronal subtypes identified using transgenic mice that express green fluorescent protein in GABAergic interneurons. J Neurosci 20:3354-3368.

Onimaru H, Homma I (2003) A novel functional neuron group for respiratory rhythm generation in the ventral medulla. J Neurosci 23:1478-1486.

Pagliardini S, Ren J, Greer JJ (2003) Ontogeny of the pre-Botzinger complex in perinatal rats. J Neurosci 23:9575-9584.

Pierani A, Moran-Rivard L, Sunshine MJ, Littman DR, Goulding M, Jessell TM (2001) Control of interneuron fate in the developing spinal cord by the progenitor homeodomain protein Dbx1. Neuron 29:367-384.

Ren J, Greer JJ (2003) Ontogeny of rhythmic motor patterns generated in the embryonic rat spinal cord. J Neurophysiol 89:1187-1195.

Shao XM, Feldman JL (1997) Respiratory rhythm generation and synaptic inhibition of expiratory neurons in pre-Botzinger complex: differential roles of glycinergic and GABAergic neural transmission. J Neurophysiol 77:1853-1860.

Sieber MA, Storm R, Martinez-de-la-Torre M, Müller T, Wende H, Reuter K, Vasyutina E, Birchmeier C (2007) Lbxl acts as a selector gene in the fate determination of somatosensory and viscerosensory relay neurons in the hindbrain. J Neurosci 27:4902-4909.

Smith JC, Greer JJ, Liu G, Feldman JL (1990) Neural mechanisms generating respiratory pattern in mammalian brainstem-spinal cord in vitro. I. Spatiotemporal patterns of motor and medullary neuron activity. J Neurophysiol 64:1149-1169.

Smith JC, Ellenberger HH, Ballanyi K, Richter DW, Feldman JL (1991) PreBötzinger complex: a brainstem region that may generate respiratory rhythm in mammals. Science 254:726-729.

Stornetta RL, Moreira TS, Takakura AC, Kang BJ, Chang DA, West GH, Brunet JF, Mulkey DK, Bayliss DA, Guyenet PG (2006) Expression of Phox $2 \mathrm{~b}$ by brainstem neurons involved in chemosensory integration in the adult rat. J Neurosci 26:10305-10314.

Thoby-Brisson M, Trinh JB, Champagnat J, Fortin G (2005) Emergence of the pre-Botzinger respiratory rhythm generator in the mouse embryo. J Neurosci 25:4307-4318.

Yvert B, Branchereau P, Meyrand P (2004) Multiple spontaneous rhythmic activity patterns generated by the embryonic mouse spinal cord occur within a specific developmental time window. J Neurophysiol 91:2101-2109.

Viemari JC, Gérard H (2002) Noradrenergic receptors and in vitro respiratory rhythm: possible interspecies differences between mouse and rat neonates. Neurosci Lett 324:149-153. 